\title{
Pratiques agricoles et eaux souterraines
}

\author{
Agriculture practices and groundwater
}

\author{
par Jean André \\ Ministère de l'Agriculture et de la Pêche \\ chef du bureau de l'eau
}

Agricultural practices affect directly groundwater resources with nitrates and pesticides. Somes experiments aiming to reduce these products are implemented in a few areas. The French governement is engaged in an assessment of the relevant public polices. This issue is facing complex stakes. No sustainable solutions will be found without the acceptance that each of us has to bear a part of responsibility.

\section{I - L'EAU ET LES ENJEUX ALIMENTAIRES}

L'agriculture est chargée d'assurer la sécurité alimentaire des pays et l'eau est un facteur-clé dans cette production. La France bénéficie d'un climat tempéré qui garantit un niveau satisfaisant de pluviométrie moyenne $700 \mathrm{~mm} / \mathrm{an}$ avec cependant des écarts marqués entre régions. En particulier le Sud de la France connaît des étés chauds et secs peu compatibles, en l'absence d'irrigation, avec de nombreuses productions agricoles, alors même que la pluviométrie annuelle est plutôt plus élevée que la moyenne nationale.

Les besoins en eau de l'agriculture sont connus. Ils représentent la moitié des consommations d'eau en France. Mais les besoins en eau des plantes qui fournissent cette production sont moins connus. Il faut qu'environ 500 litres d'eau transitent par les plantes pour obtenir $1 \mathrm{~kg}$ de farine. Ce rapport de 1/500 donne une idée des volumes d'eau nécessaires à la production agricole et explique l'importance accordée à l'irrigation dans le monde.

\section{II $\square$ LA DÉGRADATION DES EAUX SOUTERRAINES}

\section{- 2.1 L'impact de l'agriculture}

Les eaux souterraines sont alimentées à partir d'infiltration d'eau de pluie. La majeure partie du territoire français $(60 \%)$ étant constituée de terres agricoles, les activités agricoles et les pratiques culturales jouent un rôle important dans la préservation ou la dégradation des ressources en eau souterraines.

La question se présente sous les deux aspects qualitatif et quantitatif.

\subsection{Aspect quantitatif}

La grande majorité des consommations d'eau en agriculture (environ 90\%) est destinée à l'irrigation. Les prélèvements correspondants se font en partie dans des nappes souterraines. Celles-ci sont alimentées par les pluies qui tombent sur les mêmes terres qui reçoivent, en période sèche, les apports par l'irrigation.

Contrairement aux eaux de surfaces, les eaux souterraines peuvent faire l'objet d'une gestion pluriannuelle. Ce mode de gestion permet de garantir une bonne réalimentation de la ressource en limitant les prélèvements tout en introduisant de la souplesse dans les autorisations de prélèvement pour l'irrigation qui dépend des conditions climatiques.

La gestion d'une ressource souterraine se fonde d'une part sur une bonne connaissance du capital et de son évolution, et d'autre part sur le respect des autorisations de prélèvement. La nappe de Beauce est un exemple où le rapprochement entre les agriculteurs et les gestionnaires de la ressource commence à donner des résultats encourageants.

Les causes de l'augmentation des prélèvements pour l'irrigation sont la conjonction de trois facteurs : l'invention de pompes immergées de forte puissance, la valorisation économique par les exploitants de l'irrigation, le besoin de garantir une production en quantité et qualité.

La technologie des pompes immergées développée pour les forages d'alimentation en eau potable a permis d'aller chercher les eaux souterraines avec un investissement qui est intéressant pour l'agriculture. Les coûts au $\mathrm{m}^{3}$ correspondants montrent la bonne rentabilité des investissements par rapport au gain de production qu'ils procurent.

La politique agricole accorde des aides fondées sur des rendements agricoles constatés en 1992 qui prend en compte les rendements particuliers obtenus par l'irrigation. 
L'influence de cette politique sur le développement de l'irrigation est contestée par les organisations agricoles. On peut penser qu'elle n'est pas complètement neutre, mais il est difficile d'en pondérer l'impact.

L'irrigation est aussi pour l'exploitant une assurance de récolte correcte et un moyen de satisfaire les exigences de qualité. Ces dernières s'imposent de plus en plus, en particulier pour les productions sous contrats ou les productions de légumes ou de fruits.

\subsection{Aspect qualitatif}

Les activités agricoles introduisent dans les sols deux types de produits : les nitrates provenant des engrais minéraux ou des engrais organiques issus des sous-produits de l'élevage et les produits phytosanitaires.

Tous ces produits, aussi utiles qu'ils puissent être pour la croissance et la santé des plantes, sont indésirables, à partir d'une certaine concentration, dans l'eau destinée à la consommation humaine.

La politique agricole commune a contribué à l'évolution de l'utilisation des terres agricoles. La transformation de prairies en champs affecte souvent directement la qualité des eaux souterraines.

\subsubsection{Les nitrates}

Pour ce qui concerne les engrais ou amendements organiques, les nitrates constituent la principale préoccupation du fait de l'augmentation régulière des concentrations de ce produit dans les eaux souterraines et du dépassement des seuils fixés par la directive européenne. Les nitrates sont à la base de la vie et en dehors des légumineuses, ils sont irremplaçables pour garantir une récolte correcte dans le cadre socioéconomique qui s'est mis en place depuis de longues années. Il faut noter, en liaison avec le point précédant, que l'irrigation permet d'obtenir une bonne valorisation des nitrates par les plantes, alors que la culture pluviale conduit l'agriculteur à apporter un dosage correspondant à la meilleure pluie attendue. Ce surdosage non utilisé par les plantes est alors lessivé et se retrouve dans les eaux souterraines.

Dans certaines régions, les sous-produits de l'élevage représentent un volume important en équivalent nitrate qui ne peut être économiquement traité que par valorisation sur les terres.

La cohérence entre le volume des rejets et l'aptitude des terres agricoles à le recevoir est une condition préalable pour résoudre le problème. Il faut ensuite s'assurer que les épandages seront pratiqués aux bonnes périodes, ce qui nécessite la création de capacités de stockages.

C'est dans cet esprit que le Programme de maîtrise des pollutions d'origine agricole (PMPOA) a été conçu et mis en place depuis 1997. Il est encore prématuré aujourd'hui d'en attendre des résultats rapides sur les eaux souterraines compte tenu du décalage entre le début des réalisations et l'engagement de l'action mais aussi par le temps de réponse des ressources souterraines à une pollution de surface.

\subsubsection{Les produits phytosanitaires}

La lutte contre les maladies et parasites divers des plantes a conduit à l'apparition des nombreux produits phytosanitaires. Tous ces produits sont en partie lessivés par les pluies et se retrouvent dans les eaux souterraines. Certains ont mis plusieurs années pour atteindre les nappes servant à la consommation humaine. D'autres n'ont été découverts que récemment parce qu'ils n'étaient pas recherchés dans les analyses antérieures. Les nouvelles règles concernant ces produits visent à favoriser leur rétention sur les plantes ou à la surface du sol afin d'éviter la contamination par lessivage des nappes.

Contrairement aux nitrates, ces produits se trouvent en faible quantité. Il a donc fallu utiliser des méthodes de recherche très modernes et leur toxicité, même à faible dose, n'est pas connue. Ils sont néanmoins indésirables et mettent les décideurs devant un problème complexe.

Les solutions de remplacement par d'autres produits ne font souvent que reporter le problème. Le monde agricole se trouve démuni devant cette pollution des eaux souterraines par des produits autorisés. Les enjeux économiques sont très forts avec des objectifs contradictoires relatifs à la production alimentaire : développer les productions alimentaires en France et préserver la qualité des eaux de consommation.

\subsubsection{Les actions}

Face à cette dégradation de la qualité des eaux, les élus ont été conduits à prendre des décisions rapides et décisives comme l'achat de terres pour les reboiser. Ces actions énergiques affichent des résultats rapides et durables notamment lorsque la totalité ou la plus grande partie de la zone d'alimentation de la nappe devient ainsi protégée.

De son côté, la profession agricole a engagé des actions visant à rationaliser les apports de fertilisants ou de produits phytosanitaires. Le programmes "Ferti-mieux" et "Phytomieux" s'inscrivent dans cette logique de progrès. Ils ne touchent encore qu'une faible partie des exploitations, mais l'analyse des résultats permet déjà d'avoir des données sur les possibilités de restauration d'une ressource par modification des pratiques agricoles. Dans tous les cas, la réduction des intrants azotés est associée à des cultures intermédiaires qui piègent les nitrates.

\section{III — UN DILEMME SOCIO- ÉCONOMIQUE...}

La dégradation progressive de la qualité de l'eau est le résultat d'une mécanique complexe mise en œuvre par des enjeux socio-économiques. Le monde agricole considère qu'il a été guidé par d'autres acteurs économiques ou politiques puissants et refuse d'assumer seul la responsabilité de cette lente dégradation des ressources en eau.

Ce problème est d'autant plus complexe qu'il se présente sous la forme d'un dilemme entre, d'un côté une politique agricole visant à assurer la sécurité alimentaire et à conforter des structures de production rentables, et de l'autre côté le besoin plus récent de donner à l'agriculture une vocation de protection des ressources. Cette dernière option conduit à réduire, fragiliser ou changer les productions et par voie de conséquence à augmenter les aides à l'hectare ou à réduire les emplois, conséquence inévitable de la réduction de la valeur ajoutée des terres.

\section{...qui fait l'objet d'actions ponctuelles...}

Dans de nombreux départements, des expériences de réductions d'intrants agricoles sont en cours. Il est encore un peu tôt pour présenter des conclusions générales sur l'ensemble de ce sujet, sauf dans les cas où la suppression totale d'un produit polluant conduit naturellement à la restauration progres- 
sive du milieu. Mais même dans ce cas, il faut vérifier qu'il n'y a pas eu simplement substitution de produit reportant seulement le problème de quelques années, le temps d'identifier la nouvelle molécule tout aussi indésirable.

Toutefois des expériences positives ont été identifiées. Elles doivent être confirmées dans la durée et leurs conséquences socio-économiques méritent d'être approfondies.

\section{...et mérite un large débat.}

La transparence apparaît plus que jamais nécessaire dans le traitement de ce problème complexe qui ne pourra pas être réglé par les seuls processus réglementaires ni par des opérations ponctuelles financées sous la pression de forts enjeux commerciaux.

L'analyse des enjeux et des responsabilités dans les dynamiques socio-économiques et environnementales doit permettre de déboucher sur des solutions dans lesquelles chaque citoyen acceptera d'être impliqué.
En effet, il faut bien prendre conscience que toute action dans ce domaine a des répercutions qui sortent du monde agricole. Pour prendre un exemple, la réduction des intrants agricoles ou la limitation de l'irrigation conduit directement à une réduction des productions à l'hectare. Pour conserver les autres équilibres, cette contrainte va se traduire soit par une augmentation des prix agricoles ou des aides agricoles, soit par une réduction des emplois agricoles et donc une augmentation du chômage. Les conséquences socio-économiques ne sont donc pas limitées au monde agricole, mais touchent l'ensemble de la société française.

Une instance d'évaluation des politiques de préservation des ressources en eau a été créée en décembre 1999. Elle est chargée d'analyser cette question sous tous les angles. Toutes les parties prenantes dans les enjeux sur les eaux souterraines seront consultées. Dans un peu plus d'un an les conclusions de cette instance devraient proposer les voies raisonnables pour garantir la qualité de l'alimentation en eau potable des populations. 